\title{
VALIDAÇÃO DA METODOLOGIA POR CROMATAGRAFIA LÍQUIDA DE ALTA EFICIÊNCIA ACOPLADA AO DETECTOR DE ÍNDICE DE REFRAÇÃO PARA A ANÁLISE DE CARBOIDRATOS PRESENTES NO BAGAÇO DE CANA- DE- AÇÚCAR
}

\author{
A. A. FERREIRA ${ }^{1}$, E. S. P. BON ${ }^{1}$, M. M. T. CARVALHO ${ }^{1}$, D. S. PEREIRA ${ }^{1}$ \\ ${ }^{1}$ Universidade Federal do Rio de Janeiro, Instituto de Química, Departamento de Bioquímica, \\ Laboratório Bioetanol \\ E-mail para contato: aluan@ufrj.br; danielfarmacia@gmail.com
}

\begin{abstract}
RESUMO - Os biocombustíveis apareceram como uma alternativa ao combustível derivado de reservas fósseis em resposta à preocupação crescente com as questões climáticas resultantes, em grande parte, do uso do petróleo. A biomassa lignocelulósica é a maior fonte de matéria-prima para produção de etanol de segunda geração. Adicionalmente, em comparação com o uso do petróleo, os biocombustíveis tem a vantagem de serem renováveis e o seu uso tem menor impacto ambiental. A metodologia utilizada nos procedimentos analíticos para a determinação da composição química dos materiais lignocelulósicos é de extrema importância em estudos para o seu uso industrial. A técnica por CLAE usando a detecção por IR é a mais utilizada uma vez que é capaz de realizar medições rápidas, sensíveis, precisas e exatas. Para garantir a confiabilidade dessa técnica é importante que o método de análise seja validado, a fim de comprovar a garantia de qualidade e consistência dos resultados analíticos. Alguns dos parâmetros validados nesse trabalho foram a linearidade e a precisão, e ambos se mostraram satisfatórios para que a utilização do método.
\end{abstract}

\section{INTRODUÇÃO}

A incerteza associada ao fornecimento de petróleo e a necessidade de minimizar o impacto no meio ambiente têm aumentado o interesse em pesquisas em combustíveis alternativos como os biocombustíveis (Hahn-Hägerdal et al., 2006).

Os biocombustíveis surgiram como uma alternativa atraente ao combustível derivado de reservas fósseis. A biomassa lignocelulósica é a maior fonte de matéria-prima para produção de biocombustíveis de segunda geração, além disso, em comparação com os combustíveis fósseis, é renovável e não agride tanto o meio ambiente (Zhang, 2008).

Geralmente, a biomassa lignocelulósica é composta por celulose (35-50\%), hemicelulose (30-35\%) e lignina (10-25\%) (Sarkar et al., 2009). O bagaço de cana-de-açúcar, na maior parte dos países tropicais, é um dos principais materiais lignocelulósicos utilizados para a bioconversão em etanol, já que apresenta alta concentração de carboidratos, baixo custo 
de colheita, transporte e armazenagem (Pandey et al., 2000). Para liberar os carboidratos da biomassa lignocelulósica, uma etapa de pré-tratamento é necessária para expor a celulose que é, a seguir, hidrolisada utilizando enzimas fúngicas. Os monossacarídeos liberados são as potenciais fontes de carbono para a produção de etanol por meio de processos de fermentação (Kuhad et al., 2011).

A metodologia analítica utilizada para a determinação da composição química do material lignocelulósico é de extrema importância em estudos de valorização desses materiais. Deste modo, existe a necessidade de desenvolvimento de um método de análise robusto, preciso e exato para quantificação de carboidratos em amostras de biomassa, previamente hidrolisada com ácido sulfúrico (Badger, 2002).

Os métodos cromatográficos são as técnicas analíticas mais potentes para a análise de carboidratos em xaropes de biomassa lignocelulósica (Cheng et al, 2010). Cromatografia em camada delgada (CCD), cromatografia gasosa (CG) e cromatografia líquida de alta eficiência (CLAE) são comumente utilizadas para separação e identificação dos carboidratos, porém eles são frequentemente quantificados por CLAE usando a detecção por índice de refração (IR) uma vez que esse método é capaz de realizar medições rápidas, sensíveis, precisas e exatas (Raessler, 2011).

Para garantir a confiabilidade do procedimento analítico por CLAE é importante que o método de análise seja validado, a fim de comprovar a garantia de qualidade e consistência dos resultados analíticos.

\section{OBJETIVO}

Validação da metodologia analítica para a quantificação, por CLAE-IR, de cinco carboidratos (celobiose, glicose, xilose, galactose e arabinose) presentes em xaropes oriundos da hidrólise ácida do bagaço da cana-de-açúcar.

\section{MATERIAIS E MÉTODOS}

\subsection{Equipamento}

As análises dos carboidratos (celobiose, glicose, xilose, galactose e arabinose) foram realizadas no equipamento Ultimate 3000 (ThermoScientific ${ }^{\circledR}$, Germany) equipado com um detector de índice de refração RI-101 (SHODEX ${ }^{\circledR}$, Japan). O sistema de colunas utilizado é composto pelo cartucho Deashing (4.6 mm I.D. $\times 30 \mathrm{~mm}$, BioRad, USA), pré-coluna Aminex Carbo P (4.6 mm I.D. $\times 30 \mathrm{~mm}$, BioRad, USA) e coluna analítica Aminex HPX-87P $(7.8 \mathrm{~mm}$ I.D. $\times 300 \mathrm{~mm}$, BioRad, USA). O software utilizado foi o Chromeleon 6.8 (Dionex ${ }^{\circledR}$, Canada). 


\subsection{Padrões de carboidratos}

- $\quad$ D-Galactose $\left(\right.$ Merck $^{\circledR}$, teor $\left.\geq 99 \%\right)$.

- D-Xilose (Sigma ${ }^{\circledR}$, teor $\left.\geq 99 \%\right)$.

- D-Glicose $\left(\right.$ Merck $^{\circledR}$, teor $\left.\geq 99 \%\right)$.

- $\quad$ D-Arabinose $\left(\right.$ Sigma $^{\circledR}$, teor $\left.\geq 99 \%\right)$.

- $\mathrm{D}(+)$-Celobiose $\left(\right.$ Sigma $^{\circledR}$, teor $\left.\geq 99 \%\right)$.

\subsection{Condições cromatográficas}

As condições cromatográficas utilizadas foram: fase móvel: água deionizada (grau reagente tipo I com 18,2 $\mathrm{M} \Omega$-cm de resistividade, descarbonatada e filtrada em filtro de 0,2 $\mu \mathrm{m})$ com fluxo de $0,6 \mathrm{~mL}$ por minuto; temperatura do forno (apenas para coluna analítica): $80^{\circ} \mathrm{C}$; temperatura do Post-Column Cooler: $45^{\circ} \mathrm{C}$; temperatura do amostrador automático: $15^{\circ} \mathrm{C}$; temperatura do detector: $45^{\circ} \mathrm{C}$ e tempo de corrida: $22 \mathrm{~min}$.

A metodologia analítica aqui avaliada foi descrita por Sluiter et al (2008) e tem sido utilizada rotineiramente no laboratório de Bioetanol do Departamento de Bioquímica da UFRJ.

$\mathrm{Na}$ figura 1 abaixo é apresentado um cromatograma obtido por CLAE-IR de uma solução padrão com os carboidratos de interesse.

Figura 1 - Cromatograma obtido por CLAE-IR de uma solução padrão com os carboidratos de interesse.

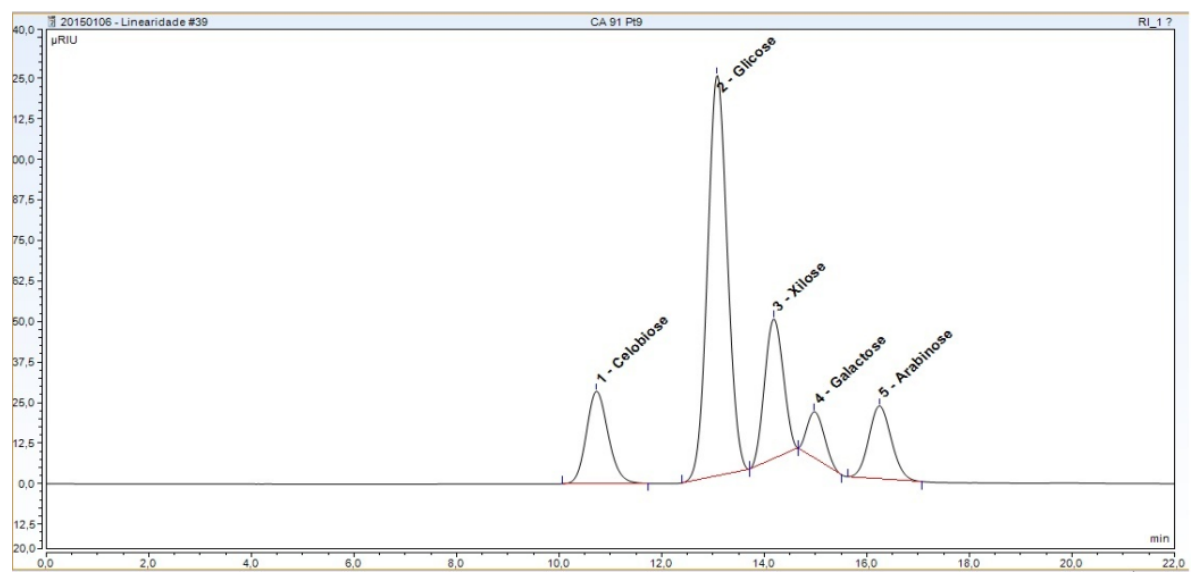




\subsection{Validação do método de caracterização}

A validação do método de caracterização foi obtida pela determinação dos seguintes parâmetros: limite de deteç̧ão, limite de quantificação, faixa de linearidade e precisão, utilizando o Programa Microsoft Office Excel 2010.

\section{RESULTADOS E DISCUSSÃO}

\subsection{Linearidade} (4):

A linearidade foi determinada pelo teste de Mandel, obtida pelas equações (1), (2), (3) e

$$
\begin{aligned}
& P G=\frac{D S^{2}}{S_{y 2}^{2}} \\
& D S^{2}=(n-2) \times S_{y / x}^{2}-(n-3) \times S_{y 2}^{2} \\
& S_{y / x}=\sqrt{\frac{\sum_{i}(y i-\hat{y} i)^{2}}{n-2}}, \text { onde } \hat{y}=\mathrm{y} * \mathrm{ax}+\mathrm{b} \\
& S_{y 2}=\sqrt{\frac{\sum_{i}(y i-\hat{y} i)^{2}}{n-3}}, \text { onde } \hat{\mathrm{y}}=\mathrm{y} * \mathrm{ax}^{2}+\mathrm{bx}+\mathrm{c}
\end{aligned}
$$

Onde:

$\mathrm{DS}^{2}=$ Diferenca de variância;

$\mathrm{S}_{\mathrm{y} / \mathrm{x}}=$ Desvio padrão dos pontos à equação da reta;

$\mathrm{S}_{\mathrm{y} 2}=$ Desvio padrão dos pontos à equação da párabola;

$\mathrm{n}=$ Número de pontos da curva

$\mathrm{y}=$ Área do pico

Na tabela 1 é apresentada a faixa linear, o coeficiente de determinação $\left(\mathrm{r}^{2}\right)$ e a equação da reta das curvas de calibração dos carboidratos. Todos os valores de $r^{2}$ foram maiores que 0,999 . 
Tabela 1 - Faixa de linearidade, $\mathrm{r}^{2}$ e equação da reta das curvas de calibração dos carboidratos

\begin{tabular}{|c|c|c|c|}
\hline Carboidrato & Faixa de linearidade (mg/mL) & $\mathbf{r}^{\mathbf{2}}$ & Equação da reta \\
\hline Celobiose & $0,20-30,15$ & 0,99998 & $\mathrm{y}=2,5085 \mathrm{x}-0,0671$ \\
\hline Glicose & $0,87-20,90$ & 0,99999 & $\mathrm{y}=2,1426 \mathrm{x}+0,0731$ \\
\hline Xilose & $0,40-9,54$ & 0,99995 & $\mathrm{y}=1,4333 \mathrm{x}+0,0408$ \\
\hline Galactose & $0,21-6,40$ & 0,99957 & $\mathrm{y}=0,7362 \mathrm{x}+0,0247$ \\
\hline Arabinose & $0,20-4,79$ & 0,99997 & $\mathrm{y}=1,9477 \mathrm{x}-0,0599$ \\
\hline
\end{tabular}

\subsection{Precisão}

A precisão dos valores de área e tempo de retenção obtidos para cada carboidrato foi determinada por múltiplas injeções $(\mathrm{n}=10)$ do menor valor de concentração de suas respectivas faixas de linearidade, analisadas no mesmo dia. Os valores de desvio padrão relativo (DPR) foram todos abaixo de 2,36\%, como pode ser visto na tabela 2 .

Tabela 2 - DPR dos valores de tempo de retenção e área para cada carboidrato

\begin{tabular}{|c|c|c|}
\hline Carboidrato & DPR do tempo de retenção (\%) & DPR da área (\%) \\
\hline Celobiose & 0,10 & 0,29 \\
\hline Glicose & 0,08 & 0,59 \\
\hline Xilose & 0,08 & 2,03 \\
\hline Galactose & 0,08 & 2,36 \\
\hline Arabinose & 0,07 & 1,87 \\
\hline
\end{tabular}

\subsection{Limite de quantificação (LDQ) e limite de deteç̧ão (LDD)}

Os limiares analíticos foram obtidos pelas equações (5) e (6), e estão descritos na tabela 3.

$$
\begin{aligned}
& L D D=\frac{3,3 s y / x}{b} \\
& L D Q=\frac{10 s y / x}{b}
\end{aligned}
$$

Onde b representa o coeficiente angular da equação da reta e $\mathrm{S}_{\mathrm{y} / \mathrm{x}}$ é o desvio padrão dos pontos à equação da reta.

Tabela 3 - Valores de LDQ e LDD dos carboidratos analisados

\begin{tabular}{|c|c|c|}
\hline Carboidrato & LDQ $(\mathbf{m g} / \mathbf{m L})$ & LDD $(\mathbf{m g} / \mathbf{m L})$ \\
\hline Celobiose & 0,58 & 0,19 \\
\hline Glicose & 0,36 & 0,12 \\
\hline Xilose & 0,22 & 0,073 \\
\hline Galactose & 0,82 & 0,27 \\
\hline
\end{tabular}




\section{CONCLUSÃO}

Validar um método significa provar e documentar resultados que indiquem que ele é seguro dentro dos limites estabelecidos e que, com sua aplicação, os resultados desejados são obtidos. Concluiu-se que o método utilizado possui uma ótima faixa de linearidade e precisão. Entretanto, observou-se que o valor de concentração mais baixo da celobiose e da galactose deve ser elevado, já que o valor de LDQ foi superior a eles. A validação desse método traz um importante avanço para as pesquisas com materiais lignocelulósicos, como a produção de bioetanol a partir do bagaço de cana de açúcar.

\section{REFERÊNCIAS}

Badger, P. C (2002). "Ethanol from cellulose: A general review." Trends in new crops and new uses $17-21$.

Cheng, C., C.-S. Chen, et al. (2010). "On-line de salting and carbohydrate analysis for immobilize denzymehydrolysis of waste cellulosic biomass by column-switching highperformance liquid chromatography." Journal of Chromatography 1217(14): 2104-2110.

Hahn-Hägerdal, B., M. Galbe, et al. (2006). "Bio-ethanol - the fuel of tomorrow from the residues of today." Trends in Biotechnology 24(12): 549-556.

Kuhad, R. C., R. Gupta, et al. (2011). "Bioethanol production from pentose sugars: Current status and future prospects." Renewable and Sustainable Energy Reviews 15 (9): 4950-4962.

Pandey, A., C. R. Soccol, et al. (2000). "Biotechnological potential of agro-industrial residues. I: sugarcane bagasse." Bioresource Technology 74 (1): 69-80.

Raessler, M. (2011). "Sample preparation and current applications of liquid chromatography for the determination of non-structural carbohydrates in plants." TrACTrends in AnalyticalChemistry 30(11): 1833-1843.

Sarkar, P., E. Bosneaga, et al. (2009). "Plant cell walls throughout evolution: towards a molecular understanding of their design principles." Journal of Experimental Botany 60(13): 3615-3635.

Sluiter, B. Hames, R. Ruiz, C. Scarlata, J. Sluiter, and D. Templeton. (2008).Determination of Sugars, Byproducts, and in Liquid Fraction Process Samples. Laboratory Analytical Procedure (LAP) of NREL. Technical Report NREL/TP-51042623.

Zhang, Y. H. P. (2008). "Reviving the carbohydrate economy via multi-product lignocellulose biorefineries." Journal of Industrial Microbiology \& Biotechnology 35(5): 367-375. 
XI Congresso Brasileiro de Engenharia Química em Iniciação Científica 